Bangl. J. Vet. Med. (2007). 5 (1 \& 2): 33-38

\title{
PRODUCTION OF FORMALIN KILLED FOWL TYPHOID VACCINE USING LOCAL ISOLATES OF SALMONELLA GALLINARUM IN BANGLADESH
}

\author{
M. G. Haider ${ }^{* 1}$, M. M. Rahman ${ }^{2}$, M. M. Hossain ${ }^{3}$, M. Rashid $^{1}$, M. A. $\operatorname{Sufian}^{1}$, M. M. Islam ${ }^{4}$ \\ and A. F. M. H. Haque ${ }^{1}$ \\ ${ }^{1}$ Livestock Research Institute, Mohakhali, Dhaka, ${ }^{2}$ Department of Medical Microbiology \& Immunology, \\ HUKM, Cheras - 5600, Kuala Lumpur, Malaysia, ${ }^{3}$ Department of Pathology, Bangladesh Agricultural \\ University, Mymensingh- 2202, ${ }^{4}$ AI Laboratory, Central Cattle Breeding and Dairy Farm, Savar, Dhaka \\ *Corresponding author's e-mail address: haidergdr@yahoo.com
}

\begin{abstract}
A locally isolated Salmonella Gallinarum (SG) was used for fowl typhoid vaccine production. $2 \times 10^{7}$ colony forming unit (CFU) of SG was used for the vaccine production. The fowl typhoid vaccine was produced with the local isolates of SG (LRI 49) strain. Efficacy study of the vaccine was performed primarily in laboratory trial in 6 weeks and then in 8 weeks old commercial layers in the field. Chickens were boasted after 4 wks of vaccination. Rapid serum plate agglutination (SPA) test and ELISA were carried out for the detection of antibody response against SG vaccinated and non-vaccinated birds. Positive results of the rapid plate agglutination and the ELISA were $81 \%$ and $77 \%$ in laboratory trial, and $76.4 \%$ and $73.8 \%$ in field trial, respectively. The chickens of vaccinated and non-vaccinated groups were challenged with the infective dose $(2 \times 2 \times$ $10^{7} \mathrm{CFU}$ ) of freshly prepared live SG bacteria and lesions were not detected in vaccinated birds at necropsy. The vaccine was proved safe and effective in terms of preventing of fowl typhoid in chickens in Bangladesh.
\end{abstract}

Key words: Fowl typhoid vaccine, potency, ELISA, plate agglutination test, Salmonella Gallinarum

\section{INTRODUCTION}

Among bacterial diseases, fowl typhoid is one of the major constraints of poultry industry in Bangladesh (Das et al., 2005). It causes heavy economic losses through mortality and reduced production (Khan et al., 1998; Hoque et al., 1997). With great expansion of the poultry rearing and farming, the disease has become wide spread problem in Bangladesh (Sarker, 1976; Rahman et al., 1979). For effective preventive and control measures of fowl typhoid, vaccination may play the vital role. But there is no locally prepared available and cost effective fowl typhoid vaccine in our country. Therefore, the present study was undertaken to the development of a fowl typhoid vaccine from the local isolates of Salmonella Gallinarum, with a view to prevent fowl typhoid in Bangladesh.

\section{MATERIALS AND METHODS}

The study was conducted at Salmonella section, Livestock Research Institute (LRI), Mohakhali, Dhaka under the Department of Livestock Services, Bangladesh and Department of Pathology, Bangladesh Agricultural University, Mymensingh during April 2005 to September 2006.

\section{Isolation and identification of Salmonellae}

\section{Collection of samples}

A total of 100 swabs from the clinical/field cases (liver, lungs, heart, spleen, intestine and ovary) of suspected chickens were collected in tetrathionate broth (TTB) in the Central Disease Investigation Laboratory (CDIL), Dhaka and Biolab, Uttra, Dhaka. 


\section{Cultivation}

All the samples were initially grown in tetrathionate broth and then on different selective and enriched media such as Salmonella -Shigella (SS) agar, lysine iron agar (LIA), triple sugar iron (TSI) agar, and brilliant green agar (BGA) to obtain pure culture (Cowan, 1985; Merchant and Packer, 1967; OIE, 2004).

\section{Morphology}

The representative Salmonellae colonies on BGA were smeared on to the slides and characterized microscopically using Gram's stain (Merchant and Packer, 1967).

\section{Biochemical test}

Five basic sugars such as dextrose, sucrose, lactose, maltose, and mannitol were used for fermentation test. In addition, TSI agar slant reaction, MR-VP test, indole test and dulcitol fermentation test were also performed (Haider et al., 2003).

\section{Motility test}

Motility test was performed according to the method described by Cowan (1985) to differentiate the motile bacteria from non-motile one (Haider et al., 2003). The identified Salmonella Gallinarum (SG) was used for the fowl typhoid vaccine production.

\section{Maintenance of stock culture}

The pure culture of SG were inoculated into the tubes containing TSI slant and incubated at $37^{0} \mathrm{C}$ for 24 hours. After the growth of organisms, the tubes were sealed with liquid paraffin (light) and kept in the refrigerator at $4^{0} \mathrm{C}$ for further studies.

\section{Determination of the dose by calculating the colony-forming unit (CFU)}

Local isolates of SG (LRI 49) were grown overnight in nutrient broth with yeast extract. After diluting nutrient broth culture, CFU of the organisms was calculated as per standard procedure (OIE, 2004). The result of CFU was expressed as the number of organisms/ml of sample.

\section{Fowl typhoid vaccine production}

Locally isolated Salmonella Gallinarum (LRI 49) was used for the production of formalin-killed vaccine. Master seed was prepared from the local isolates of $S$. Gallinarum (LRI 49). The bulk culture of the bacteria were made using shaker incubator in $1000 \mathrm{ml}$ container. Then the formalin was added in per litter of broth culture and incubated for 24 hours in room temperature. The broth culture was cheeked by culturing in BGA, SS agar and TSI agar slant and no growth was found. The $\mathrm{pH}$ was checked and aluminium hydroxide gel was added as adjuvant, and incubated at $37^{\circ} \mathrm{C}$ for 24 hours. As per the procedure of Biological Standardization (Walker, 1999; OIE, 2004) inactivated fowl typhoid vaccine was prepared and aliquoted in a volume of $100 \mathrm{ml}$ in glass bottle and preserved at $4-8^{0} \mathrm{C}$.

\section{Vaccination and potency test}

In laboratory trails, 100 Isa Brown healthy chickens, 6 weeks of age, were taken from a commercial layer farm serologically free from SG. A volume of $0.5 \mathrm{ml}\left(2 \times 10^{7} \mathrm{CFU}\right)$ vaccine/bird was injected intramuscularly. After 28 days, the vaccinated chickens were boosted with same dose and 2 weeks after boosting sera were collected for SPA and ELISA test. Sera were also collected from non-vaccinated birds at similar time schedule of vaccinated birds. Rapid serum plate agglutination and ELISA test were carried out with the sera to determine the potency of the vaccine. After 4 weeks of immunization the birds was challenged with infective dose $\left(2 \times 2 \times 10^{7}\right.$ $\mathrm{CFU}$ ) of live bacteria. The challenged birds were examined to detect clinical signs. Two weeks after challenge postmortem examination was done for the detection of gross lesions. For field trial, five commercial layer flocks (flock no. 1, 2 3, 4 \& 5) were vaccinated subcutaneously with the early mentioned dose and one flock (flock no. 6) was treated as a control during trial. Each flock contained 1000 birds, 8 weeks old Shaver White chickens. The vaccinated chickens were boosted with same dose after 28 days. 
Fowl typhoid vaccine

\section{Rapid serum plate agglutination (SPA) test}

Standard Salmonella (Nobilis ${ }^{\circledR}$ SP) antigen manufactured by Intervet International, Holland was used for SPA test. $0.02 \mathrm{ml}$ antigen and $0.02 \mathrm{ml}$ chicken sera were placed side by side with a micropipette on a glass slide and mixed thoroughly by stirring with tooth pick followed by rocking. Results were read within 2 minutes.

\section{ELISA test}

The serum samples of vaccinated and control birds were subjected to determine the antibody titre. FLOCKSCREEN $^{\mathrm{TM}}$ Salmonella antibody ELISA kit (Guild Hay Ltd. UK) was used. A 550 was measured using an ELISA reader (Toso Ltd. MRP-A4i, Tokyo, Japan) and the OD values were plotted as per manufacturer instruction. For the validity of the ELISA test, mean negative control absorbance was $<0.2$ and mean positive control absorbance was at least 2.5 times the mean negative absorbance. ELISA titer values of the samples that showed $<450$, 451- 1500, and $>1501$ were interpreted as negative, suspect and positive, respectively.

\section{RESULTS AND DISCUSSION}

\section{Isolation and identification of S. Gallinarum (SG)}

The colony characters of SG in SS agar was whitish or slight grayish in appearance with dark central spot reflecting production of hydrogen sulfide on the media. In LIA, the SG produced slight blackish colonies, in BGA pink white colonies, and in TSI agar slant alkaline reaction (red colour) was observed. In Gram's staining, the bacteria appeared as small rod, gram-negative and single or paired in arrangement. All the isolates fermented glucose and manitol but did not ferment lactose and sucrose. Some Salmonellae (88.24\%) fermented maltose and some (11.76\%) did not. All of the isolates were MR positive but VP and indole were negative. Motility test is fundamental basis for the detection of motile and non-motile Salmonella organisms. Non-motile organisms were considered to be either $S$. pullorum or $S$. Gallinarum. The motile organisms were considered as other species of Salmonella. In the present study, 3 isolates were non motile and 14 were motile.

The ability or inability of Salmonellae to ferment different carbohydrates was considered as fundamental basis for their identification but species identification was difficult. In the present study 2 out of 3 isolated non-motile Salmonellae fermented dulcitol. On the basis of this test these two isolated bacteria were grouped into Salmonella Gallinarum. Finally different biochemical tests were used and a total of 17 Salmonellae was isolated and characterized in this study. Out of 17 isolates, one Salmonella was characterized as Salmonella Pullorum (SP), 2 Salmonella Gallinarum (SG) and 14 other motile Salmonellae. These isolates were also confirmed as SG using PCR and RFLP methods in the Department of Pathology. The PCR and RFLP results did not quoted here. However, one local isolate of SG was used for the vaccine production against fowl typhoid.

\section{Efficacy and potency of fowl typhoid vaccine in laboratory trials}

The antibody responses of vaccinated chickens were shown in Table 1 and Table 2. Swelling in the muscle was found in $3 \%$ chickens due to intramuscular route of vaccination. Then the vaccinated and non-vaccinated chickens were challenged with infective dose and slaughtered after 14 days of post-challenge. No clinical signs were observed and no gross lesions were found after necropsy in vaccinated birds.

Table 1. Detection of antibody response of chickens in laboratory trials by ELISA test

\begin{tabular}{|ll|lll|}
\hline No. of birds & No. of sera tested & ELISA & & \\
\hline \multirow{2}{*}{100} & \multirow{2}{*}{92} & Negative $(<450)$ & Suspected (451- 1500) & Positive $(>1501)$ \\
\cline { 2 - 5 } & & 0 & 15 & 77 \\
\hline
\end{tabular}

In ELISA evaluation, sera that showed <450, 451- 1500, and > 1501 were considered as negative, suspected and positive, respectively.

Table 2. Detection of antibody response of chickens in laboratory trials by SPA test

\begin{tabular}{|ll|lll|}
\hline No. of birds & No. of sera tested & SPA & & \\
\hline \multirow{2}{*}{100} & \multirow{2}{*}{100} & + & ++ & +++ \\
\cline { 2 - 5 } & & 08 & 11 & 81 \\
\hline
\end{tabular}

In SPA (Nobilis ${ }^{\circledR}$ SP antigen) evaluation, sera that showed aggregation within one minute on a plate were considered as positive. 


\section{Efficacy of fowl typhoid vaccine in commercial layer flocks in the field}

The immune responses of vaccinated (flock no. 1, 2, 3, 4 \& 5) and non-vaccinated chickens (flock no. 6) were given in the Table 3 and 4 . Subcutaneous route was the better and safe for the vaccination. No skin lesion was found at the site of vaccination.

Table 3. Detection of antibody response of commercial layer flocks in the field trials by ELISA test

\begin{tabular}{|ll|lll|}
\hline No. of flock & No. of sera tested & ELISA & & \\
\cline { 3 - 5 } & & Negative $(>450)$ & Suspect (451- 1500) & Positive $(>1501)$ \\
\hline 1 & 92 & 00 & 18 & 74 \\
2 & 92 & 00 & 14 & 78 \\
3 & 92 & 00 & 21 & 71 \\
4 & 92 & 00 & 17 & 75 \\
5 & 92 & 00 & 21 & 71 \\
6 & 92 & 92 & 00 & 00 \\
\hline
\end{tabular}

In ELISA evaluation, sera that showed $<450,451-1500$, and $>1501$ were considered as negative, suspect and positive, respectively.

Table 4. Detection of antibody response of commercial layer flocks in the field trials by SPA test

\begin{tabular}{|ll|lll|}
\hline No. of flock & No. of sera tested & SPA & ++ & +++ \\
\cline { 3 - 5 } & & + & 13 & 76 \\
\hline 1 & 100 & 11 & 11 & 79 \\
3 & 100 & 10 & 15 & 73 \\
4 & 100 & 12 & 12 & 78 \\
5 & 100 & 11 & 13 & 76 \\
6 & 100 & 00 & 00 & 00 \\
\hline
\end{tabular}

In SPA (Nobilis ${ }^{\circledR}$ SP antigen) evaluation, sera that showed aggregation within one minute on a plate were considered as positive.

The colony characters of Salmonella Gallinarum (SG) in SS agar, LIA, TSI agar and BGA were corresponded with Perez et al. (2004), Sharma and Katock (1996), Yuno et al. (1995) and Old (1990). In Gram's staining, the morphology of the isolated bacteria was small rod shape, Gram negative and single or paired in arrangement that is supported by Freeman, (1985). All of the isolates fermented glucose and mannitol but did not ferment lactose and sucrose. Some Salmonellae fermented maltose and some did not. All of the isolates were MR positive but VP and indole were negative. These findings were previously suggested by a number of scientists (Merchant and Packer, 1967; Buxton and Fraser, 1977; Freeman, 1985; Sujatha et al., 2003; Khan et al., 1998). Non-motile organisms were considered to be either $S$. Pullorum or $S$. Gallinarum. The motile organisms were considered as others species of Salmonella (OIE, 2004). In the present study 2 out of 3 isolated non-motile Salmonellae fermented dulcitol. On the basis of this test these two isolated bacteria were grouped into Salmonella Gallinarum (OIE, 2004).

The efficacy of vaccines from field trials is very limited. The purpose of this field study was to give more information about the additional effect of vaccination under field circumstances in a situation with a certain field standardized biosecurity program (SBP). This field trail was carried out on the basis of several assumptions. All vaccinated flocks were placed on farms with a certified SBP because a maximum effect of vaccination was found by good hygienic measurement (Fris et al., 1993). Finally the vaccinated and non-vaccinated flocks were monitored serologically.

Properly serological testing hypothesis of the effect of vaccination, however, the vaccinated and nonvaccinated flock must be comparable with respect to other risk factors including housing, husbandry, feeding, management, etc. In this study, influence of these risk factors did not justify. 
In this study, the positive titer of ELISA and SPA test results were $77 \%$ and $81 \%$, respectively in laboratory and field trials. The positive titer of ELISA and SPA test results were $73.8 \%$ and $76.4 \%$, respectively in laboratory and field trials after vaccination. The results are similar with the work of other investigators who have demonstrated that subcutaneous administration of SG-9R vaccine gives variable protection against experimental challenge of fowl typhoid (Silva et al., 1980). Better immune response of fowl typhoid was observed because it was used considering the SBP. The same findings were reported by Silva et al. (1980). One of the greatest advantages reported of the use of $9 \mathrm{R}$ vaccine is that it gives good protection and it does not interfere with tests used for pullorum- typhoid control because it does not produce agglutination titers (Espinosa et al., 1975).

The antibody level of this study was somewhat lower than the other authors (Feberwee et al., 2000a). The lower antibody level was speculated due to the environmental factors and the problem of electricity for preserving the serum. The results of potency test after challenge in this study were better than the results of Fris et al., 1993. The vaccine also gives more protection than Salmonella Enteritidis vaccine (Feberwee et al., 2000b). The vaccine was more effective than the Salmonella Harder vaccine (Rolan et al., 2004). The vaccine would be more potent in Bangladesh than the commercially available FT vaccine because it was produced by the locally isolated Salmonella Gallinarum.

Fowl typhoid is a major problem in the poultry industry in Bangladesh. It causes great production losses in every year. The control and prevention of fowl typhoid in poultry stock and the economic distress for poultry farmers are arguments of introducing a vaccination program. Other aspects like biosecurity, however, are also important when discussing the implementation of vaccine as a tool in Salmonella control. Besides efficacy of a vaccine, political and emotional reasons, these arguments are also important when considering implementation of vaccination programs in the organized fowl typhoid control programme.

\section{ACKNOWLEDGEMENTS}

The authors are grateful to The Ministry of Fisheries and Livestock, Government of The Peoples Republic of Bangladesh sanctioning the project "Production of Vaccines for Prevention of Diseases of Livestock and Poultry” under the Department of Livestock Services, Bangladesh.

\section{REFERENCES}

1. Buxton A and Fraser G (1977). Animal Microbiology. Vol. 1. Blackwell Scientific Publications, Oxford, London, Edinburgh, Melbourne. pp. 103-115.

2. Cowan ST (1985). Cowan and Steel's Manual for the Identification of Medical Bacteria. $2^{\text {nd }}$ edn., Cambridge University Press, Cambridge, UK.

3. Das PM, Rajib DMM, Noor M and Islam MR (2005). Retrospective analysis on the proportional incidence of poultry diseases in greater Mymensingh district of Bangladesh. In proceeding of $4^{\text {th }}$ International Poultry Show and Seminar, from February 28 to March 2, 2005, held in Bangladesh China Friendship Conference Centre, Agargaon. Dhaka. Pp 3539.

4. Espinosa JE, Flores R and Pijoan C (1975). Evalucion de la vacuna 9R liofilizada para prevenir la infection por Salmonella Gallinarum. Tec.Pecu. Mex. 29: 50-53.

5. Feberwee A, de Vries TS, Elbers ARW and de Jong WA (2000b). Results of a Salmonella enteritidis Vaccination Field Trial in Broiler Breeder Flocks in The Netherlands. Avian Diseases 44: 249-255.

6. Feberwee A, de Vries TS, Hartman EG, de Wit JJ, Elbers ARW and de Jong WA (2000a). Vaccination against Salmonella Gallinarum 9R strain: Evaluation of efficacy, Safety, and Performance of Serologic Salmonella Tests. Avian Diseases 45: 83-91.

7. Freeman BA (1985). Burrow's Text Book of Microbiology. $22^{\text {nd }}$ edn., W. B. Saunders Company, London, UK. pp. 372472.

8. Fris C, Van den Bosch J and Van Dit Marsch (1993). A retrospective case. Control study of risk factors associated with Salmonella enterica sub sp. enterica serovar Enteritidis on Dutch broiler breeder farms. Avian Pathology 24: 255-272.

9. Haider MG, Hossain MG, Hossain MS, Chowdhury EH, Das PM and Hossain MM (2003). Isolation and characterization of enterobacteria associated with health and disease in Sonali chickens. Bangladesh Journal Veterinary Medicine 2: 15-21.

10. Hoque MM, Biswas HR and Rahman L (1997). Isolation, identification and production of Salmonella pullorum colored antigen in Bangladesh for the rapid whole blood test. Asian-Australasian Journal of Animal Sciences 10: 141-146.

11. Khan AHMNA, Bari ASM, Islam MR, Das PM and Ali MY (1998). Pullorum disease in semi mature chickens and its experimental pathology. Bangladesh Veterinary Journal 32: 124-128. 
12. Merchant IA and Packer RA (1967). Veterinary Bacteriology and Virology. $7^{\text {th }}$ edn., The Iowa State University Press, Ames, Iowa, USA. pp. 211-306.

13. OIE (Office International Des Epizooties) (2004). Salmonellosis and fowl typhoid \& pullorum diseases. In: OIE Guide2. Manual of Diagnostic tests and vaccines for terrestrial Animals.

14. Old DC (1990). Salmonella. In: Topley \& Wilson's Principles of Bacteriology, Virology and Immunity. $8^{\text {th }}$ edn., Parker, M. T. and Duerden, B. I. (eds.). Vol. 2 Systematic Bacteriology. Edward Arnold. A division of Hodder \& Stoughton, London, UK. pp. 470-475.

15. Perez C, Rivera S, Pirela A, Rincon H, Mavarez Y and Roman R (2004). Isolation of Salmonella in poultry carcasses and evaluation of the effectiveness of different enrichment and selective media. Revista Cientifica Facultad Ciencias Veterinarias Universidad del Zulia 14: 177-185.

16. Rahman MM, Chowdhury TIMF, Rahman MM and Hossain WIMA (1979). Survillance of Salmonella \& Escherichia organisms in poultry feed. Bangladesh Veterinary Journal 15: 59-62.

17. Rolan K, Tingi S, Waruer E and Size more D (2004). Comparision of Different Attenuation Strategies in Development of a Salmonella harder vaccine. Avian Diseases 48: 445-452.

18. Sarker AJ (1976). The prevalence of avian diseases in Bangladesh Agricultural University poultry farm. Bangladesh Veterinary Journal 10: 64-66.

19. Sharma M and Katock RC (1996). Deadly outbreak in chicks owing to Salmonella typhimurium. Indian Journal of Poultry Science 31: 60-62.

20. Silva EN, Snoeyenbos GH, Weinack OM and Smyser CF (1980). Studies on the use of 9R strain of Salmonella Gallinarum as a vaccine in chickens. Avian Diseases 25: 38-52.

21. Sujatha K, Dhanalakshmi K and Rao AS (2003). Isolation and characterisation of Salmonella Gallinarum from chicken. Indian Veterinary Journal 80: 473-474.

22. Walker PD (1999). FAO Vaccine Manual. Daya Publishing House, Delhi. pp. 44-59.

23. Yuno MML, Terzolo HR, Fernandez HD, Malena RC and Altuna ME (1995). Evaluation of selective culture media for isolation of Salmonella from poultry. Revista Argentina de Microbiology 27: 57-69. 